Review Article

\title{
Biological and Clinical Effects of Calciprotein Particles on Chronic Kidney Disease-Mineral and Bone Disorder
}

\author{
Kenichi Akiyama, ${ }^{1}$ Takaaki Kimura, ${ }^{2}$ and Kazuhiro Shiizaki $\mathbb{D}^{1,3}$ \\ ${ }^{1}$ Division of Anti-Ageing Medicine, Center for Molecular Medicine, Jichi Medical University, Shimotsuke, Tochigi, Japan \\ ${ }^{2}$ Division of Renal Surgery and Transplantation, Department of Urology, Jichi Medical University, Shimotsuke, Tochigi, Japan \\ ${ }^{3}$ Division of Nephrology, Department of Internal Medicine, Jichi Medical University, Shimotsuke, Tochigi, Japan
}

Correspondence should be addressed to Kazuhiro Shiizaki; shiizaki@jichi.ac.jp

Received 6 December 2017; Revised 24 January 2018; Accepted 12 February 2018; Published 27 March 2018

Academic Editor: Michael Horowitz

Copyright (C) 2018 Kenichi Akiyama et al. This is an open access article distributed under the Creative Commons Attribution License, which permits unrestricted use, distribution, and reproduction in any medium, provided the original work is properly cited.

\begin{abstract}
Calciprotein particles (CPPs) are a new biological marker of chronic kidney disease-mineral and bone disorder (CKD-MBD). CPPs consist of phosphate, calcium, and some proteins, with phosphate being the major contributor to the level and biological activity of CPPs. Recent studies have shown the physiological and pathological significance of CPPs, including contributions to bone and mineral metabolism, and to tissue and organ impairments such as cardiovascular damage and inflammatory responses. These actions are well known as important aspects of CKD-MBD. Fibroblast growth factor 23 (FGF23), which is secreted from the bone as the phosphaturic hormone, is markedly elevated in CKD-MBD. Many clinical studies have shown significant relationships between the level of FGF23 and outcomes such as mortality, prevalence of cardiovascular disease, bone fracture, and levels of inflammatory markers. Basic and clinical studies have suggested that CPPs contribute to synthesis and secretion of FGF23. Surgical treatments such as renal transplantation and parathyroidectomy for patients with CKD-MBD suppress excess levels of phosphate, calcium, parathyroid hormone (PTH), and FGF23, which are related to the CPP level. Therefore, suppression of CPPs might also contribute to improved clinical outcomes after these treatments.
\end{abstract}

\section{Introduction}

Chronic kidney disease (CKD) has a high risk of complication with cardiovascular disorders, even before end-stage $\mathrm{CKD}$ requiring dialysis therapy. A major cause is soft tissue calcification due to impaired calcium (Ca) and phosphorus (P) metabolism, which is referred to as CKD-MBD (CKDmineral and bone disorder). Homeostasis of bone mineral metabolism collapses with the decline of renal function, but various compensation mechanisms maintain blood $\mathrm{Ca}$ and $\mathrm{P}$ levels for protection against systemic organ damage in early-stage CKD. When the estimated glomerular filtration rate (eGFR) declines to about $60 \mathrm{~mL} / \mathrm{min} / 1.73 \mathrm{~m}^{2}$, the active vitamin $\mathrm{D}\left(1,25(\mathrm{OH})_{2} \mathrm{D}_{3}\right)$ level starts to decline and parathyroid hormone $(\mathrm{PTH})$ subsequently elevates. However, the fibroblast growth factor 23 (FGF23) level elevates earlier than these hormonal changes. Since the expression of Klotho, a coreceptor of FGF23, declines in the kidney in very earlystage CKD, FGF23 rises due to resistance to FGF23 signaling in the kidney [1]. FGF23 can increase the urinary phosphorus excretion rate $(\mathrm{FEP}=$ (urinary phosphorus/blood phosphorus)/(urinary creatinine/blood creatinine) $\times 100(\%)$ ) as a phosphaturic factor and suppress activation of vitamin $\mathrm{D}$, resulting in the major trigger in the course of CKD-MBD (Figure 1) [2].

Calciprotein particles (CPPs) are a complex of $\mathrm{Ca}, \mathrm{P}$, and proteins such as fetuin-A that have the major physiological function of transport of hydroxyapatite to the bone without crystallization in other tissues. The blood level of CPPs elevates with excess $\mathrm{P}$ and $\mathrm{Ca}$, and this contributes to arteriosclerosis [3] and inflammatory response [4] in CKD. The CPP level increases in the G1 and G2 stages of CKD, just before the rise of FGF23 [3]. These clinical findings lead to the hypothesis that CPPs might induce FGF23, and this is 


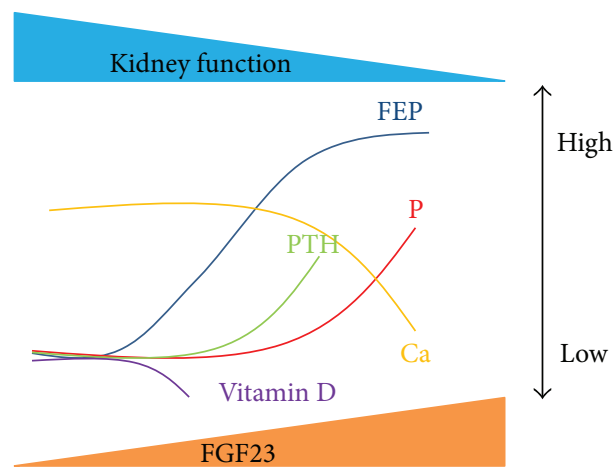

FIGURE 1: Changes of CKD-MBD-related markers accompanying decline of renal function. FEP and FGF23 levels show correlated elevation from the early to middle stages of $\mathrm{CKD}$, with a subsequent decrease of $1,25(\mathrm{OH})_{2} \mathrm{D}_{3}$ and increase of PTH. When FEP cannot respond to the high level of FGF23 in end-stage CKD, the $\mathrm{P}$ and $\mathrm{Ca}$ levels start to elevate and decline, respectively.

supported by basic studies, as described below. We recently also confirmed this hypothesis in vitro and in vivo [5]. In addition, the CPP level correlates significantly with the levels of $\mathrm{P}$ and FGF23, but not $\mathrm{Ca}$, and thus can rise even in a state of hyperphosphatemia and hypocalcemia associated with progression of CKD [6]. However, a further study is necessary to establish the mechanism of CPP formation in vivo. Sequential changes in CKD-MBD might involve CPPs and FGF23 for homeostasis of bone and mineral metabolism.

\section{Physiology and Pathology of CPPs}

2.1. Components of CPPs. CPPs consisting of mineral and fetuin-A and matrix GLA protein (Mgp) at 18\%, 80\%, and $2 \%$, respectively, were first discovered in the blood of rats treated with alendronate [7]. Since Mgp is a protein with autocrine and paracrine effects, $\mathrm{Ca}, \mathrm{P}$, and fetuin- $\mathrm{A}$ are thought to be the major components of CPPs. Physiologically, CPPs transport hydroxyapatite material to the teeth and bones for growth and remodeling, without precipitating this material in soft tissues [8]. Fetuin-A, which is mainly produced in hepatocytes, is a glycoprotein of about $60 \mathrm{kDa}$ that is abundant in extracellular fluid and has a concentration in the blood of $0.5-1.0 \mathrm{~g} / \mathrm{L}$ in healthy people. Inactivation of the insulin receptor tyrosine kinase and transforming growth factor $\beta$ pathways are well-known biological effects of fetuin$A$, and severe ectopic calcification was found in fetuin-Adeficient mice $[9,10]$.

In vitro analysis showed that initial (primary) CPPs with diameter $60-75 \mathrm{~nm}$ are complexes of fetuin-A and a Posner cluster of $\mathrm{Ca}_{9}\left(\mathrm{PO}_{4}\right)_{6}$, which is the minimum unit of calcium phosphate (diameter $9.5 \AA$ ) that exists as a colloid. Developed (secondary) CPPs include hydroxyapatite resulting from calcium phosphate crystallization over time in the core and are about $120-150 \mathrm{~nm}$ in size $[11,12]$. The strong negative charges in the $\beta$ sheet structure of the cystatin-like D1 domain of fetuin-A are thought to promote binding with calcium phosphate [11].
2.2. Metabolism of CPPs. The half-lives of primary and secondary CPPs are 149 and $45 \mathrm{~min}$, respectively, in mice intravenously injected with fluorescently labeled synthetic CPPs. The particles are phagocytosed by F4/80- or CD68-positive macrophages and Kupffer cells in the liver and by macrophage receptor with collagenous structure- (MARCO-) positive macrophages in the spleen. Primary CPPs are also deposited in the liver, kidney, and bone marrow, and so it is assumed that CPPs are metabolized in these organs [13]. CPPs might be metabolized for physiological protection against organ damage resulting from their accumulation. Similar organ damage such as cardiovascular disorders occurs with excess production of CPPs in CKD-MBD and with the loss of fetuin-A, as described below.

2.3. Involvement of CPPs in Inflammatory Response and Cardiovascular Disorders. Only secondary CPPs induced cell necrosis, mineralization, and TNF- $\alpha$ mRNA and protein in experiments in primary human aortic vascular smooth muscle cells (VSMCs) treated with primary or secondary CPPs. These effects were exacerbated and attenuated by the administration of TNF- $\alpha$ and its inhibitor, respectively. These findings suggest that secondary CPPs might locally induce cytokines such as TNF- $\alpha$, which may result in vascular damage [14]. Treatment with an inhibitor of CPP formation ameliorated vascular damage in rats fed an adeninecontaining diet and reduced elevation of the blood CPP level and vascular calcification [15]. On the other hand, secondary CPPs were found in vascular lesions, in which CD68-positive macrophages phagocytosed secondary CPPs through scavenger receptor-A (SR-A) I/II in mice deficient in ApoE and fetuin-A [13]. Therefore, CPPs might be contributing to vascular calcification in these pathological animal models.

2.4. CPPs Induce FGF23 in Bone. FGF23 is a hormone that promotes $\mathrm{P}$ excretion in urine by suppressing type II sodium-dependent phosphate cotransporters (Npt2a and $\mathrm{Npt} 2 \mathrm{c}$ ), which are expressed on the luminal side of proximal renal tubular cells in the kidney. The blood FGF23 level elevates according to the balance of $\mathrm{P}$ for its homeostasis. In rat osteoblast-like cells (UMR-106) and mouse osteocytelike cells (IDG-SW3), P in the medium upregulates FGF23 mRNA in a dose-dependent manner $[16,17]$. However, a low Ca level suppresses this upregulation [5]. This finding was also confirmed in an animal model treated with a high phosphate diet [18]. Moreover, experiments in which blood $\mathrm{Ca}$ and $\mathrm{P}$ levels were controlled in mice lacking one or both genes for the Ca-sensing receptor and $\mathrm{PTH}$ revealed that the product of the $\mathrm{Ca}$ and $\mathrm{P}$ levels is the strongest factor regulating the blood FGF23 level [19].

FGF23 is composed of a signal sequence (24 amino acids), N-terminal core homology domain (155 amino acids), and a C-terminal domain (72 amino acids). It is inactivated by cleavage at the sequence of 176RXXR179, which is recognized by a proteolytic enzyme. Intact FGF23 and C-terminal FGF23 assays that measure only full-length and both fulllength and C-terminal fragments, respectively, are available. Many clinical studies of CKD have shown a significant correlation of these assays with each other and with the elevation 


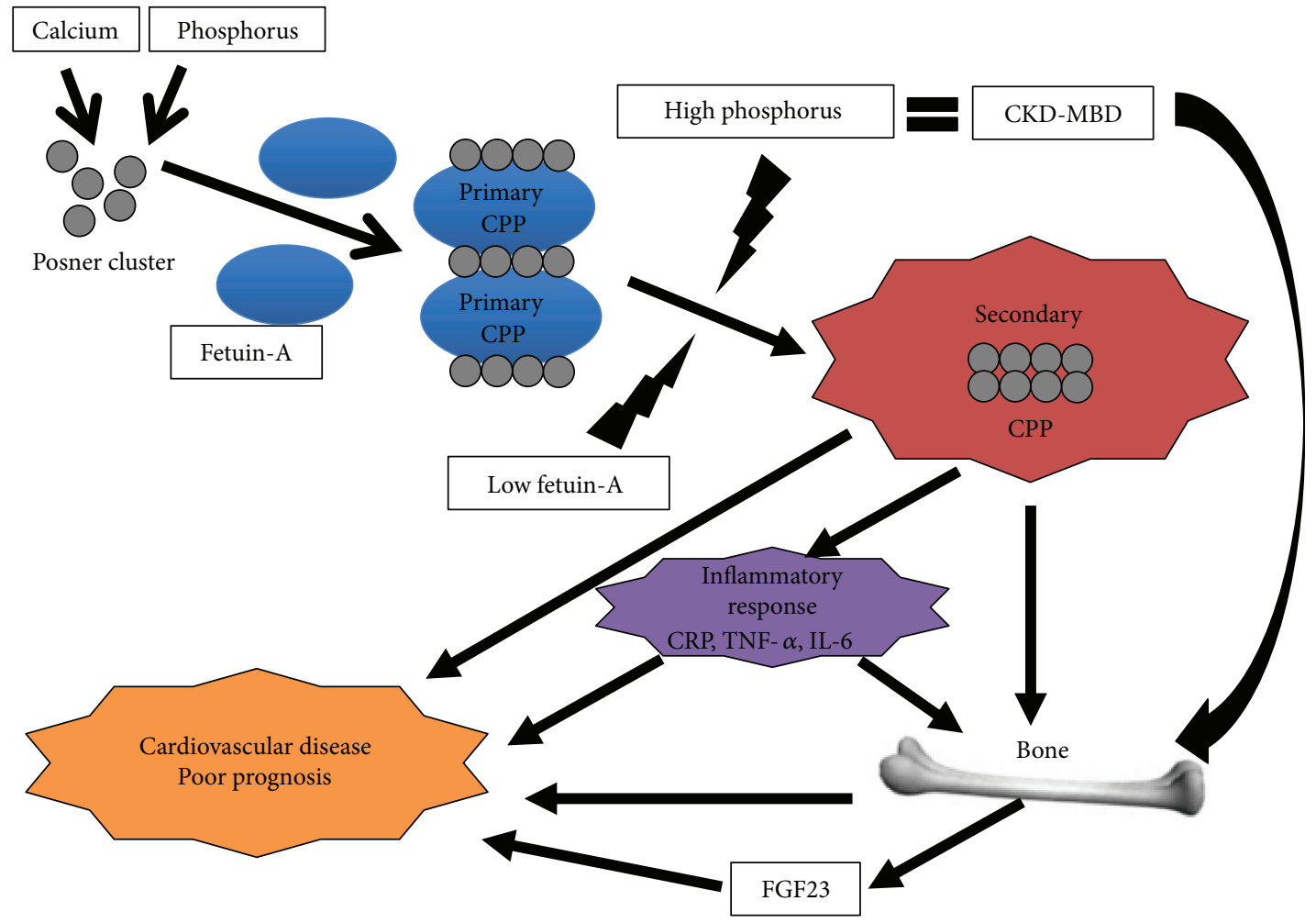

FIGURE 2: The physiological role of primary CPPs is accurate transportation of materials for the bones and teeth. Developed (secondary) CPPs may induce an inflammatory response through induction of TNF- $\alpha$, IL-6, and CRP in CKD and may upregulate FGF23 in bone cells. These sequential abnormalities can contribute to the risk of cardiovascular disease and the poor prognosis in patients with CKD.

of $\mathrm{P}$ in the blood and progression of $\mathrm{CKD}$, indicating the similar clinical significance of the two assays [20]. The blood CPP level is also significantly correlated with intact FGF23 and P levels in dialysis and nondialysis patients with CKD [6].

These basic and clinical findings suggest that CPPs (the complex of $\mathrm{Ca}$ and $\mathrm{P}$ ), but not $\mathrm{P}$ itself, might be a physiological and pathological contributor to P-induced FGF23. However, it will be necessary to evaluate the in vivo effects of inhibitors of CPP formation on P-induced FGF23 to confirm this hypothesis. TNF- $\alpha$ and IL- $1 \beta$ elevated FGF2 3 mRNA and protein levels in IDG-SW3 cells [21], and similar findings were obtained following the administration of secondary CPPs [22]. Therefore, CPPs may induce inflammatory responses that both result in organ damage, including cardiovascular disease, and induce FGF23 in bone cells.

\section{Clinical Significance of CPPs}

The blood CPP level, the high sensitivity C-reactive protein (CRP) level, the coronary artery calcification score, and the aortic pulse wave velocity (aPWV) are all elevated with loss of renal function and are all correlated with each other $[3,4]$, while the blood fetuin-A level is negatively correlated with blood CRP, all-cause mortality, and cardiovascular death in patients with hemodialysis [23]. Therefore, consumptive loss of fetuin-A caused by excess CPP formation and inhibition of production of fetuin- $A$ in the liver by uremic toxins such as indoxyl sulfate might also contribute to the high mortality in these patients [24].

A significant relationship between the blood CPP level and mortality has recently been shown in patients with CKD, and new CPP assays have been developed. The turbidity method using a nephelometer system has been applied for the evaluation of CPP status in serum, based on the measurement of the half time for maximal transition (T50) from primary to secondary CPPs in serum after adding $\mathrm{Ca}$ and $\mathrm{P}$ solutions. The T50 was significantly shorter in patients with hemodialysis than in healthy volunteers, and the T50 in fetuin-A-deficient mice was also shorter than that in wildtype controls [12]. A recent study also suggested that secondary CPPs are easily formed from precursors such as primary CPPs in CKD and that this might contribute to tissue and organ damage under stress in CKD [6]. T50 shortening is related to the high risk of death in patients in CKD stages G3 and G4 and in kidney transplant recipients $[25,26]$ and to all-cause death, myocardial infarction, hospitalization due to unstable angina pectoris, heart failure, and peripheral vascular disease in patients on hemodialysis [27].

Methods for measuring primary and secondary CPPs in the blood by flow cytometry using fluorescently labeled probes have also been developed [28]. The secondary CPP level in the blood is significantly higher in hemodialysis patients than in healthy subjects, and P, FGF23, IL-1 $\beta$, IL-6, and diabetes mellitus are significantly correlated with this level. These results suggest that secondary CPPs are a 
TABLE 1: The effects of surgical treatments on the major complications of CKD-MBD.

\begin{tabular}{|c|c|c|c|c|c|}
\hline & \multicolumn{3}{|c|}{ Relations of biochemical parameters } & \multicolumn{2}{|c|}{ Effects of surgical treatments } \\
\hline & Calcium & Phosphorus & FGF23 & Renal transplantation & Parathyroidectomy \\
\hline All-cause mortality & Positive [47] & Positive [47] & Positive [31] & Improved [33] & Improved [40] \\
\hline Cardiovascular events & Positive [48] & Positive [48] & Positive [31] & Improved [34] & Improved [41] \\
\hline Vascular calcification & Positive [49] & Positive [49] & Positive [31] & & Improved [42] \\
\hline Cardiac hypertrophy & Positive [50] & Positive [51] & Positive [31] & Improved [35] & Improved [43] \\
\hline Anemia & Positive [52] & Positive [52] & Positive [53] & & Improved [44] \\
\hline Malnutrition & & & Positive [31] & Improved [36] & Improved [45] \\
\hline Chronic inflammation & Positive [54] & Positive [54] & Positive [54] & Improved [37] & Improved [46] \\
\hline
\end{tabular}

new biomarker for the pathological condition of CKDMBD (Figure 2).

\section{CKD-MBD Normalization by Surgery May Improve Clinical Outcomes (Table 1)}

The major surgical treatments for CKD-MBD are kidney transplantation and parathyroidectomy. Both exert powerful effects that are not obtained in conventional dialysis or drug treatment, resulting in significant improvement of CKDMBD. Since the CPP level is significantly correlated with the P level in patients with CKD [6], CPP formation may be suppressed following parathyroidectomy and kidney transplantation, which might markedly decrease the P level. However, further studies on the actual change in the CPP level after surgery are required.

The $\mathrm{P}$ level markedly decreases because the recovery of kidney function activates phosphaturic function following kidney transplantation in patients with end-stage renal failure. Most recipients can quickly withdraw from dialysis therapy, except in a case of delayed graft function. The $\mathrm{Ca}$ level also decreases immediately after kidney transplantation and reaches a normal level after about six months. Along with the recovery of active vitamin D synthesis in the grafted kidney, the Ca status is improved. However, high levels of PTH and FGF23 often persist, as described below [29], and a high PTH level in patients with advanced hyperplasia of the parathyroid gland, such as nodular hyperplasia, is present even years after renal transplantation [30].

The extremely high FGF23 level in patients with CKD$\mathrm{MBD}$ is associated with all-cause mortality, risk of cardiovascular events, vascular calcification, cardiac hypertrophy, anemia, malnutrition, and chronic inflammation [31]. The FGF23 level starts to decrease about one week after transplantation but may not reach the normal range for almost one year [32]. However, renal transplantation improves most of the complications associated with the high level of FGF23 [33-37].

The rapid decrease in excess PTH after parathyroidectomy causes hypocalcemia and hypophosphatemia because of the rapid flow of $\mathrm{Ca}$ and $\mathrm{P}$ into the bone due to marked suppression of high turnover bone (hungry bone syndrome) [38]. Excess FGF23 also decreases [39], and these biochemical changes indicate a decreased CPP level after parathyroidectomy.
Many reports have shown that parathyroidectomy improves clinical outcomes such as all-cause mortality, cardiovascular events, vascular calcification, cardiac hypertrophy, anemia, malnutrition, and chronic inflammation [40-46]. Improved outcomes by kidney transplantation and parathyroidectomy are related to the improvement of CKDMBD and particularly normalization of excess P, FGF23, and PTH. These effects may all contribute to the suppression of the secondary CPP formation, as a protective mechanism for inhibiting aggressive inflammatory activity [16].

\section{Conclusion}

CPPs have attracted recent attention in clinical and basic research as a new molecular marker involved in the pathology of CKD-MBD. Excess P intake from original phosphorusrich food and from fast food, soft drinks, and food preservatives might enhance CPP formation and result in a poor prognosis for patients with CKD. The new findings on CPPs have improved the understanding of the pathophysiology of CKD-MBD and assist in the management of CKD-MBD for the prevention of complications such as cardiovascular disease. Surgical interventions may be most effective for improving clinical outcomes through amelioration of CKDMBD, including reduction of the levels of FGF23 and CPPs.

\section{Conflicts of Interest}

The authors declare that there is no conflict of interest regarding this article.

\section{References}

[1] M. C. Hu, K. Shiizaki, M. Kuro-o, and O. W. Moe, "Fibroblast growth factor 23 and Klotho: physiology and pathophysiology of an endocrine network of mineral metabolism," Annual Review of Physiology, vol. 75, no. 1, pp. 503-533, 2013.

[2] T. Kimura, K. Shiiizaki, M. Kuro-o, and T. Yagisawa, "FGF23 initially plays an important role on phosphate homeostasis in chronic kidney disease," Journal of the American Society Nephrology, vol. 27, article 221A, 2016.

[3] T. Hamano, I. Matsui, S. Mikami et al., "Fetuin-mineral complex reflects extraosseous calcification stress in CKD," Journal of the American Society of Nephrology, vol. 21, no. 11, pp. 1998-2007, 2010.

[4] E. R. Smith, M. L. Ford, L. A. Tomlinson, C. Rajkumar, L. P. McMahon, and S. G. Holt, "Phosphorylated fetuin-A- 
containing calciprotein particles are associated with aortic stiffness and a procalcific milieu in patients with pre-dialysis CKD," Nephrology, Dialysis, Transplantation, vol. 27, no. 5, pp. 1957-1966, 2012.

[5] K. Akiyama, "Calciprotein particle contributes to the synthesis and secretion of fibroblast growth factor 23 induced by dietary phosphate intake," Journal of the American Society Nephrology, vol. 28, p. 210, 2017.

[6] Y. Miura, Y. Iwazu, K. Shiizaki et al., "Identification and quantification of plasma calciprotein particles with distinct physical properties in patients with chronic kidney disease," Scientific Reports, vol. 8, no. 1, p. 1256, 2017.

[7] P. A. Price, G. R. Thomas, A. W. Pardini, W. F. Figueira, J. M. Caputo, and M. K. Williamson, "Discovery of a high molecular weight complex of calcium, phosphate, fetuin, and matrix $\gamma$-carboxyglutamic acid protein in the serum of etidronatetreated rats," Journal of Biological Chemistry, vol. 277, no. 6, pp. 3926-3934, 2002.

[8] T. Schinke, C. Amendt, A. Trindl, O. Pöschke, W. MüllerEsterl, and W. Jahnen-Dechent, "The serum protein $\alpha_{2}$-HS glycoprotein/fetuin inhibits apatite formation in vitro and in mineralizing calvaria cells. A possible role in mineralization and calcium homeostasis," Journal of Biological Chemistry, vol. 271, no. 34, pp. 20789-20796, 1996.

[9] W. Jahnen-Dechent, T. Schinke, A. Trindl et al., "Cloning and targeted deletion of the mouse fetuin gene," Journal of Biological Chemistry, vol. 272, no. 50, pp. 31496-31503, 1997.

[10] C. Schafer, A. Heiss, A. Schwarz et al., "The serum protein $\alpha_{2}$-Heremans-Schmid glycoprotein/fetuin-A is a systemically acting inhibitor of ectopic calcification," The Journal of Clinical Investigation, vol. 112, no. 3, pp. 357-366, 2003.

[11] A. Heiss, A. DuChesne, B. Denecke et al., "Structural basis of calcification inhibition by $\alpha_{2}$-HS glycoprotein/fetuin-A. Formation of colloidal calciprotein particles," Journal of Biological Chemistry, vol. 278, no. 15, pp. 13333-13341, 2003.

[12] A. Pasch, S. Farese, S. Graber et al., "Nanoparticle-based test measures overall propensity for calcification in serum," Journal of the American Society Nephrology, vol. 23, no. 10, pp. 1744-1752, 2012.

[13] M. Herrmann, C. Schäfer, A. Heiss et al., "Clearance of fetuinA-containing calciprotein particles is mediated by scavenger receptor-A," Circulation Research, vol. 111, no. 5, pp. 575584, 2012.

[14] P. Aghagolzadeh, M. Bachtler, R. Bijarnia et al., "Calcification of vascular smooth muscle cells is induced by secondary calciprotein particles and enhanced by tumor necrosis factor- $\alpha$," Atherosclerosis, vol. 251, pp. 404-414, 2016.

[15] I. Matsui, T. Hamano, S. Mikami et al., "Fully phosphorylated fetuin-A forms a mineral complex in the serum of rats with adenine-induced renal failure," Kidney International, vol. 75, no. 9, pp. 915-928, 2009.

[16] M. Hori, Y. Kinoshita, M. Taguchi, and S. Fukumoto, "Phosphate enhances Fgf23 expression through reactive oxygen species in UMR-106 cells," Journal of Bone and Mineral Metabolism, vol. 34, no. 2, pp. 132-139, 2016.

[17] N. Ito, D. M. Findlay, P. H. Anderson, L. F. Bonewald, and G. J. Atkins, "Extracellular phosphate modulates the effect of $1 \alpha$, 25-dihydroxy vitamin $\mathrm{D}_{3}(1,25 \mathrm{D})$ on osteocyte like cells," The Journal of Steroid Biochemistry and Molecular Biology, vol. 136, pp. 183-186, 2013.
[18] M. E. Rodriguez-Ortiz, I. Lopez, J. R. Muñoz-Castañeda et al., "Calcium deficiency reduces circulating levels of FGF23," Journal of the American Society of Nephrology, vol. 23, no. 7, pp. 1190-1197, 2012.

[19] S. J. Quinn, A. R. Thomsen, J. L. Pang et al., "Interactions between calcium and phosphorus in the regulation of the production of fibroblast growth factor 23 in vivo," American Journal of Physiology Endocrinology and Metabolism, vol. 304, no. 3, pp. E310-E320, 2013.

[20] D. Fliser, B. Kollerits, U. Neyer et al., "Fibroblast growth factor 23 (FGF23) predicts progression of chronic kidney disease: the mild to moderate kidney disease (MMKD) study," Journal of the American Society Nephrology, vol. 18, no. 9, pp. 26002608, 2007.

[21] N. Ito, A. R. Wijenayaka, M. Prideaux et al., "Regulation of FGF23 expression in IDG-SW3 osteocytes and human bone by pro-inflammatory stimuli," Molecular and Cellular Endocrinology, vol. 399, pp. 208-218, 2015.

[22] J. M. Rutkowski, J. Pastor, K. Sun et al., "Adiponectin alters renal calcium and phosphate excretion through regulation of klotho expression," Kidney International, vol. 91, no. 2, pp. 324-337, 2017.

[23] M. Ketteler, P. Bongartz, R. Westenfeld et al., "Association of low fetuin-A (AHSG) concentrations in serum with cardiovascular mortality in patients on dialysis: a cross-sectional study," The Lancet, vol. 361, no. 9360, pp. 827-833, 2003.

[24] A. Ochi, K. Mori, S. Nakatani et al., "Indoxyl sulfate suppresses hepatic fetuin-A expression via the aryl hydrocarbon receptor in HepG2 cells," Nephrology, Dialysis, Transplantation, vol. 30, no. 10, pp. 1683-1692, 2015.

[25] E. R. Smith, M. L. Ford, L. A. Tomlinson et al., "Serum calcification propensity predicts all-cause mortality in predialysis CKD," Journal of the American Society Nephrology, vol. 25, no. 2, pp. 339-348, 2014.

[26] C. A. Keyzer, M. H. de Borst, E. van den Berg et al., "Calcification propensity and survival among renal transplant recipients," Journal of the American Society of Nephrology, vol. 27, no. 1, pp. 239-248, 2016.

[27] A. Pash, G. A. Block, M. Bachtler et al., "Blood calcification propensity, cardiovascular events, and survival in patients receiving hemodialysis in the EVOLVE trial," Clinical Journal of the American Society of Nephrology, vol. 12, no. 2, pp. 315322, 2017.

[28] E. R. Smith, T. D. Hewitson, M. M. X. Cai et al., "A novel fluorescent probe-based flow cytometric assay for mineralcontaining nanoparticles in serum," Scientific Reports, vol. 7, no. 1, article 5686, 2017.

[29] S. M. Sprague, V. Belozeroff, M. D. Danese, L. P. Martin, and K. Olgaard, "Abnormal bone and mineral metabolism in kidney transplant patients-a review," American Journal of Nephrology, vol. 28, no. 2, pp. 246-253, 2008.

[30] A. M. D'Alessandro, J. S. Melzer, J. D. Pirsch et al., "Tertiary hyperparathyroidism after renal transplantation: operative indications," Surgery, vol. 106, no. 6, pp. 10491055, 1989.

[31] X. Lu and M. C. Hu, "Klotho/FGF23 axis in chronic kidney disease and cardiovascular disease," Kidney Disease, vol. 3, no. 1, pp. 15-23, 2017.

[32] P. Evenepoel, B. K. Meijers, H. de Jonge et al., "Recovery of hyperphosphatoninism and renal phosphorus wasting one year after successful renal transplantation," Clinical Journal 
of the American Society of Nephrology, vol. 3, no. 6, pp. 18291836, 2008.

[33] R. A. Wolfe, V. B. Ashby, E. L. Milford et al., "Comparison of mortality in all patients on dialysis, patients on dialysis awaiting transplantation, and recipients of a first cadaveric transplant," The New England Journal of Medicine, vol. 341, no. 23, pp. 1725-1730, 1999.

[34] H. Pilmore, H. Dent, S. Chang, S. P. McDonald, and S. J. Chadban, "Reduction in cardiovascular death after kidney transplantation," Transplantation, vol. 89, no. 7, pp. 851857, 2010.

[35] B. Hewing, A. M. Dehn, O. Staeck et al., "Improved left ventricular structure and function after successful kidney transplantation," Kidney \& Blood Pressure Research, vol. 41, no. 5, pp. 701-709, 2016.

[36] S. Beckmann, N. Ivanovic, G. Drent, T. Ruppar, and S. De Geest, "Weight gain, overweight and obesity in solid organ transplantation - a study protocol for a systematic literature review," Systematic Reviews, vol. 4, no. 1, p. 2, 2015.

[37] M. Z. Molnar, K. Nagy, A. Remport et al., "Inflammatory markers and outcomes in kidney transplant recipients," Transplantation, vol. 101, no. 9, pp. 2152-2164, 2017.

[38] N. Jain and R. F. Reilly, "Hungry bone syndrome," Current Opinion in Nephrology and Hypertension, vol. 26, no. 4, pp. 250-255, 2017.

[39] S. C. Liao, S. H. Moi, F. F. Chou, C. H. Yang, and J. B. Chen, "Changes in serum concentrations of fibroblast growth factor 23 and soluble klotho in hemodialysis patients after total parathyroidectomy," BioMed Research International, vol. 2016, Article ID 6453803, 6 pages, 2016.

[40] H. Komaba, M. Taniguchi, A. Wada, K. Iseki, Y. Tsubakihara, and M. Fukagawa, "Parathyroidectomy and survival among Japanese hemodialysis patients with secondary hyperparathyroidism," Kidney International, vol. 88, no. 2, pp. 350-359, 2015.

[41] M. Nagashima, K. Hashimoto, T. Shinsato et al., "Marked improvement of left ventricular function after parathyroidectomy in a hemodialysis patient with secondary hyperparathyroidism and left ventricular dysfunction," Circulation Journal, vol. 67, no. 3, pp. 269-272, 2003.

[42] A. J. Bleyer, J. Burkart, M. Piazza, G. Russell, M. Rohr, and J. J. Carr, "Changes in cardiovascular calcification after parathyroidectomy in patients with ESRD," American Journal of Kidney Diseases, vol. 46, no. 3, pp. 464-469, 2005.

[43] T. Drueke, M. Fauchet, J. Fleury et al., "Effect of parathyroidectomy on left-ventricular function in haemodialysis patients," The Lancet, vol. 315, no. 8160, pp. 112-114, 1980.

[44] J. A. Trunzo, C. R. McHenry, J. A. Schulak, and S. M. Wilhelm, "Effect of parathyroidectomy on anemia and erythropoietin dosing in end-stage renal disease patients with hyperparathyroidism," Surgery, vol. 144, no. 6, pp. 915-8; discussion 919, 2008.

[45] F. F. Chou, C. H. Lee, and C. T. Lee, "Muscle force and bone mineral density after parathyroidectomy and subcutaneous autotransplantation for secondary hyperparathyroidism," World Journal of Surgery, vol. 23, no. 5, pp. 452-457, 1999, discussion 456-7.

[46] C. Yasunaga, M. Nakamoto, K. Matsuo, G. Nishihara, T. Yoshida, and T. Goya, "Effects of a parathyroidectomy on the immune system and nutritional condition in chronic dialysis patients with secondary hyperparathyroidism," The
American Journal of Surgery, vol. 178, no. 4, pp. 332-336, 1999.

[47] G. A. Block, P. S. Klassen, J. M. Lazarus, N. Ofsthun, E. G. Lowrie, and G. M. Chertow, "Mineral metabolism, mortality, and morbidity in maintenance hemodialysis," Journal of the American Society Nephrology, vol. 15, no. 8, pp. 2208-2218, 2004.

[48] Y. Slinin, R. N. Foley, and A. J. Collins, "Calcium, phosphorus, parathyroid hormone, and cardiovascular disease in hemodialysis patients: the USRDS waves 1, 3, and 4 study," Journal of the American Society Nephrology, vol. 16, no. 6, pp. 17881793, 2005.

[49] S. M. More and N. X. Chen, "Mechanisms of vascular calcification in chronic kidney disease," Journal of American Society of Nephrology, vol. 19, no. 2, pp. 213-216, 2008.

[50] J. Li, N. Wu, Y. Li, K. Ye, M. He, and R. Hu, "Cross-sectional analysis of serum calcium levels for associations with left ventricular hypertrophy in normocalcemia individuals with type 2 diabetes," Cardiovascular Diabetology, vol. 14, no. 1, p. 43, 2015.

[51] J. Zou, Y. Yu, P. Wu et al., "Serum phosphorus is related to left ventricular remodeling independent of renal function in hospitalized patients with chronic kidney disease," International Journal of Cardiology, vol. 221, pp. 134-140, 2016.

[52] M. Boronat, A. Santana, E. Bosch, D. Lorenzo, M. Riaño, and C. García-Cantón, "Relationship between anemia and serum concentrations of calcium and phosphorus in advanced non-dialysis-dependent chronic kidney disease," Nephron, vol. 135, no. 2, pp. 97-104, 2017.

[53] L. M. Coe, S. V. Madathil, C. Casu, B. Lanske, S. Rivella, and D. Sitara, "FGF-23 is a negative regulator of prenatal and postnatal erythropoiesis," Journal of Biological Chemistry, vol. 289, no. 14, pp. 9795-9810, 2014.

[54] C. Francis and V. David, "Inflammation regulates fibroblast growth factor 23 production," Current Opinion in Nephrology and Hypertension, vol. 25, no. 4, pp. 325-332, 2016. 


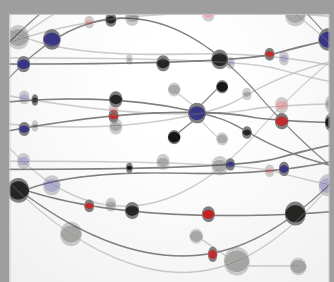

The Scientific World Journal
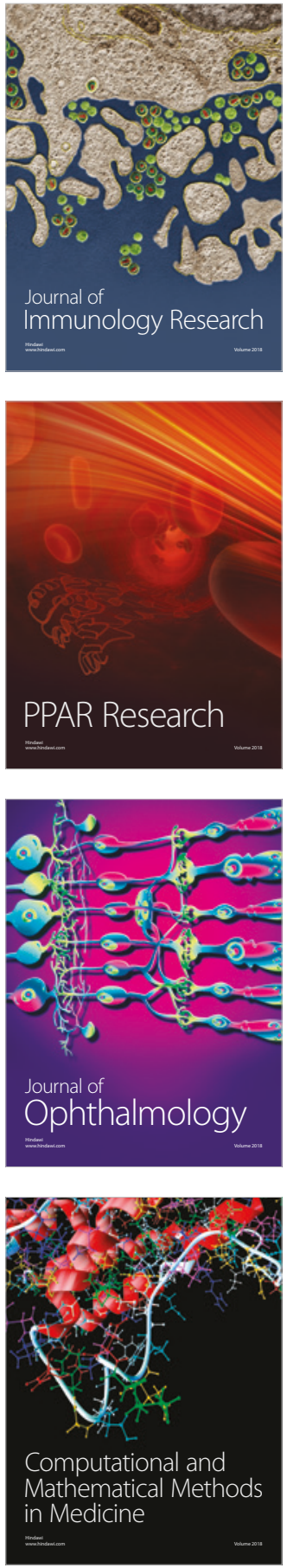

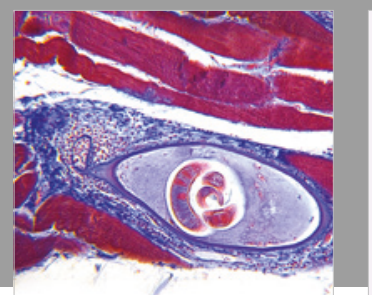

Gastroenterology Research and Practice

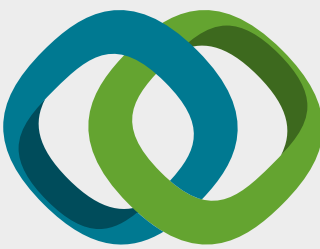

\section{Hindawi}

Submit your manuscripts at

www.hindawi.com
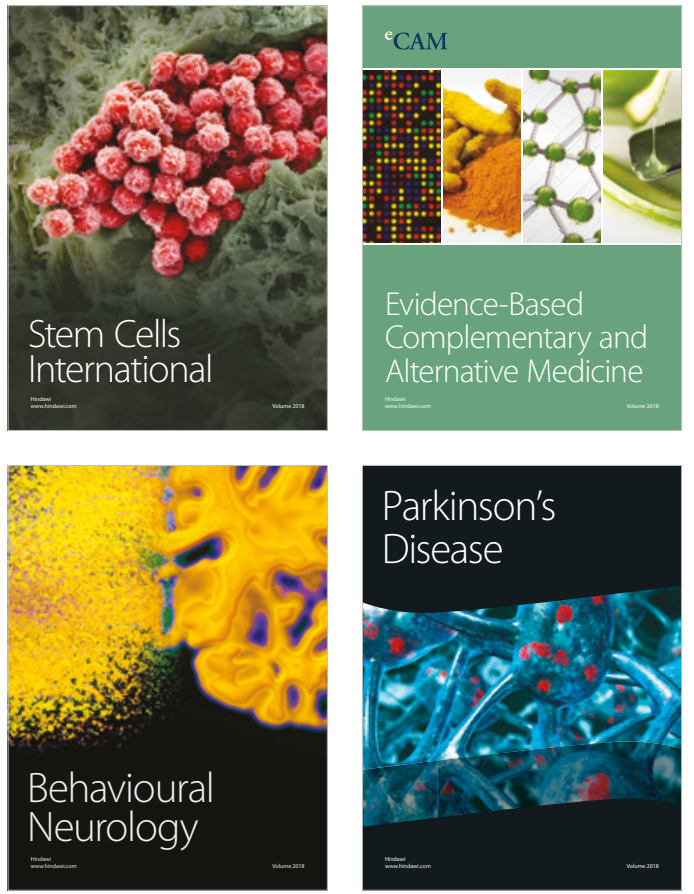

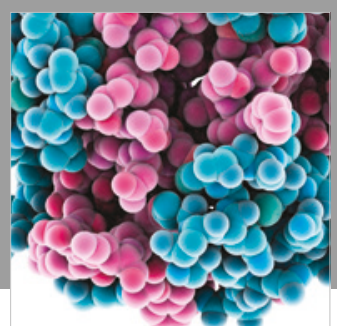

ournal of

Diabetes Research

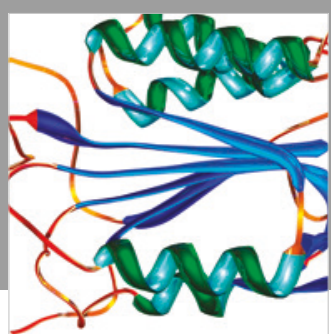

Disease Markers
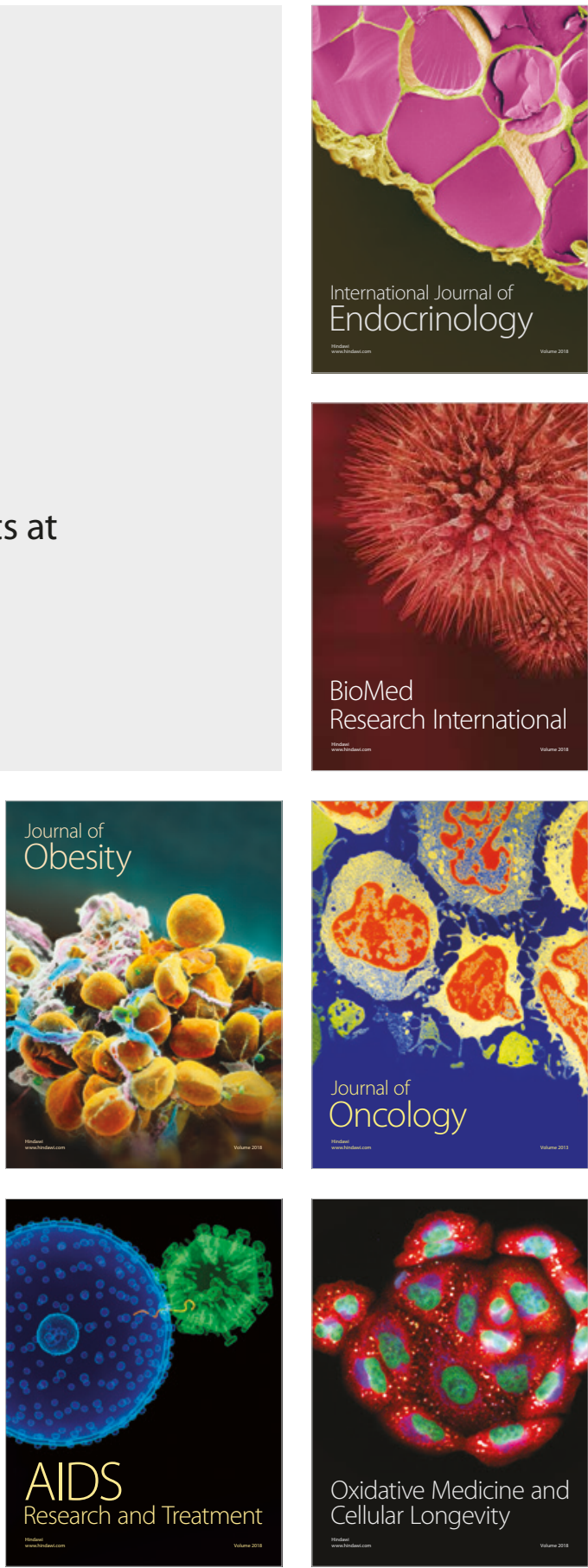